\title{
Wideband Dispersion Compensation in Hexagonal Lattice Photonic Crystal Fiber
}

\author{
Esmat Jafari and Mohammad Ali Mansouri-Birjandi* \\ Faculty of Electrical and Computer Engineering, University of Sistan and Baluchestan, \\ Zahedan, Iran
}

*Corresponding Author Email: mansouri@ece.usb.ac.ir

Received: Mar. 16, 2017, Revised: May. 17, 2017, Accepted: Jun. 12, 2017, Available Online: Dec. 1, 2018

DOI: 10.29252/ijop.12.2.91

\begin{abstract}
In this paper, a new structure is provided for the dispersion compensating photonic crystal fibers in order to broaden the chromatic dispersion and increase the dispersion compensating capability in a wide wavelength range. In the structure, putting elliptical holes in the first ring of the inner core clad of a dispersion compensating fiber of the hexagonal lattice, increases the wavelength range of the dispersion compensation, and causes this fiber to have the capability of dispersion compensation in the whole $E$ to $U$ telecommunication bands. In this fiber, the minimal dispersion will be $-1006 \mathrm{ps} /(\mathrm{nm} . \mathrm{km})$ at the $1.68 \mu \mathrm{m}$ wavelength and at the $1.55 \mu \mathrm{m}$ wavelength the dispersion coefficient will be $710 \mathrm{ps} /(\mathbf{n m} . \mathrm{km})$. The simulations are all done using the finite difference time domain numerical method.
\end{abstract}

KeywORDS: Photonic Crystal Fiber, Dispersion Compensation, Chromatic Dispersion, Negative Dispersion Coefficient.

\section{I.INTRODUCTION}

Every structure in which the refractive index changes periodically is called photonic crystal. So far, many optical devices have been designed based on photonic crystals such as optical fibers [1], [2]. The photonic crystal fibers have microscopic arrays of air channels that help tuning the dispersion and control the confinement loss in a way that is impossible in an ordinary fiber [3], [4]. The chromatic dispersion of the optical fibers is a key parameter that causes an optical pulse to become broadened in an optical communication system [5]. Therefore, dispersion compensation and dispersion compensation methods are of the important issues in long-distance transmissions and need considerable attention. On the other hand, in the recent years, photonic crystal fibers have been of significant use due to their unique characteristics. Based on that, dispersion compensating photonic crystal fibers have been of great importance. Dispersion compensating photonic crystal fibers have two cores; one inner core at the center of the fiber, and one outer core supporting two super modes [6], [7]. The mode coupling between the inner core and the outer core ring causes a negative dispersion in the fiber. The second core can be created in the fiber in many ways, like reducing the diameters of the air holes in one of the rings [8], [9] or using another material in a ring [10], [11] or removing one ring of the air holes [12], that all ends in an increase in the refractive index in the ring.

Using the genetic algorithm, Fujisawa et al. introduced a hexagonal lattice photonic crystal fiber for dispersion compensation in band $\mathrm{C}$ [13]. Matsui et al. introduced a photonic crystal fiber capable of dispersion compensation in three telecommunication bands [14]. The dispersion amplitude of this photonic crystal fiber is $-100 \mathrm{ps} /(\mathrm{nm} . \mathrm{km})$ which is known to be low. Therefore, to compensate the accumulated dispersion of the transmission fiber, a long dispersion compensating fiber should be used and this increases the costs. Selim Habib et al. introduced a hexagonal lattice wideband dispersion compensating fiber [15] that the 
minimum dispersion is near the $1.59 \mu \mathrm{m}$ wavelength.

This paper tends to present a hexagonal lattice wideband dispersion compensating fiber. In this structure, placing elliptical holes in the first ring of the fiber inner core clad increases the dispersion compensation wavelength ranges and makes the fiber capable of dispersion compensation throughout the $\mathrm{E}$ to $\mathrm{U}$ telecommunication bands comprising 5 telecommunication bands. The proposed structure in this paper, compared to the other similar structures presented earlier, includes a wider wavelength range and also has a more negative dispersion coefficient compared to the one presented by Matsui et al.

In the second section, the way the dispersion is calculated will be discussed briefly and then the geometry of the proposed structure will be introduced. In the third section, the simulation and the results obtained using the finite difference time domain numerical method will be shown. The fourth part will be the conclusion.

\section{TheORY AND INTRODUCING THE Proposed Structure}

There exist different kinds of dispersion. The most important factor of dispersion in the single-mode fibers is the chromatic dispersion which is calculated with the Eq. (1):

$D_{C}=\frac{1}{L} \frac{\Delta t_{C}}{\Delta \lambda}=-\frac{\lambda}{c} \frac{d^{2} n_{e f f}}{d \lambda^{2}}$

where $D_{c}, \Delta t_{c}$ and $\Delta \lambda$ are the chromatic dispersion coefficient, chromatic dispersion and the spectral width. $L$ is the fiber length, $\lambda$ is the wavelength in vacuum, $c$ is the speed of light in vacuum and $n_{\text {eff }}$ is the effective index of the fiber [16].

The structure presented in this article in Fig. 1 is a hexagonal lattice wideband dispersion compensating fiber. This fiber has two ring of the inner clad and two rings of the outer clad. Instead of the circular holes in the first ring of the inner core clad, elliptical holes will be placed and the outer core is created through a reduction in the diameter of the air holes in the third ring. The optimum parameters of the structure are presented in Table 1. The background material is pure silica.

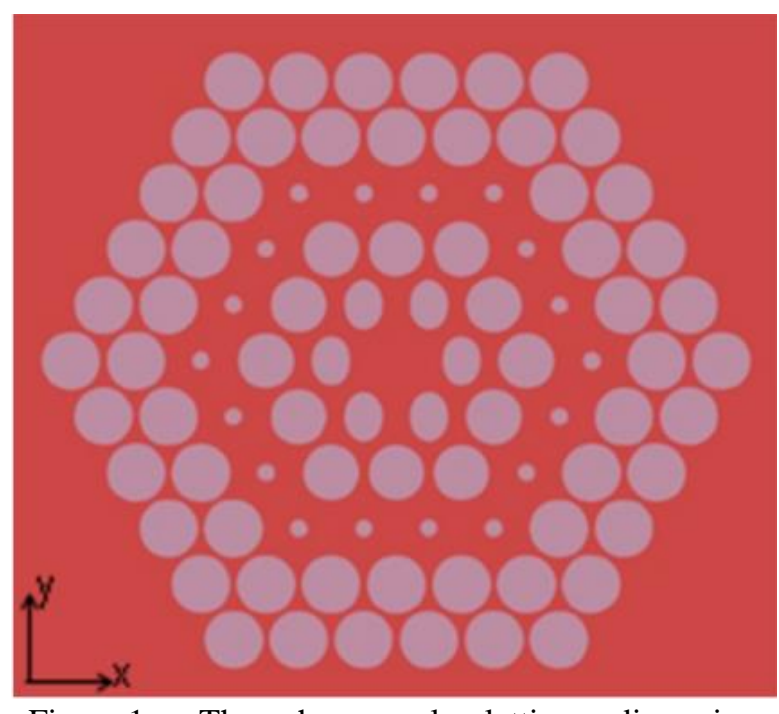

Fig. 1. The hexagonal lattice dispersion compensating fiber with elliptical holes.

Table 1. The quantities of the parameters of the proposed structure

\begin{tabular}{lc}
\hline \hline Parameter & Value $(\mu \mathrm{m})$ \\
\hline \hline Pitch of lattice $(\Lambda)$ & 1.00 \\
Air hole diameter of inner core clad $\left(\mathrm{d}_{1}\right)$ & 0.84 \\
Air hole diameter of outer core $\left(\mathrm{d}_{2}\right)$ & 0.34 \\
Air hole diameter of outer core clad $\left(\mathrm{d}_{3}\right)$ & 0.90 \\
The diameter of the ellipse in X axis $\left(\mathrm{d}_{\mathrm{x}}\right)$ & 0.29 \\
The diameter of the ellipse in Y axis $\left(\mathrm{d}_{\mathrm{y}}\right)$ & 0.40 \\
\hline \hline
\end{tabular}

To calculate the dispersion profile of the structure, the finite difference time domain numerical method is used. The numerical methods in analyzing the photonic crystal fiber can be divided in two categories of time domain and frequency domain. The numerical methods in the time domain show less variety compared to the other methods but they are more compatible with parallel processing and the information extracted through the simulation of the time domain are given in a wide range of frequencies.

\section{III.SIMULATIONS AND RESULTS}

In order for the influence of the proposed structure on the broadening of the chromatic 
dispersion profile to be properly observable, first, a hexagonal lattice simple dispersion compensating fiber is simulated. Then, the elliptical holes are placed in the first ring of the inner core clad and the new structure dispersion profile is calculated.

Figure 2 shows the structure of the simple hexagonal lattice wideband dispersion compensating fiber. The characteristics and parameters are presented in Table 2.

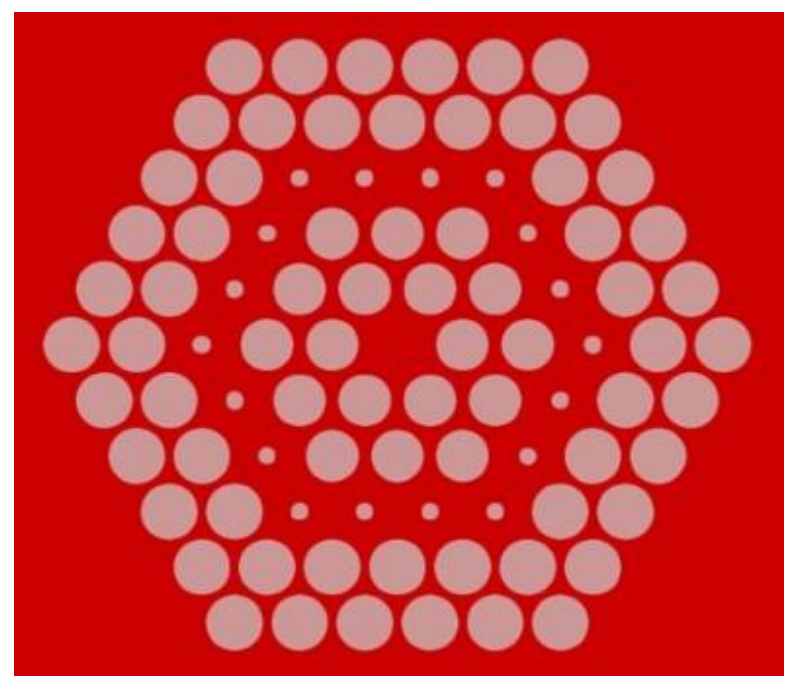

Fig. 2. The simple hexagonal lattice wideband dispersion compensating fiber.

Table 2. The parameters of the simple hexagonal lattice wideband dispersion compensating fiber.

\begin{tabular}{lc}
\hline \hline Parameter & Value $(\mu \mathrm{m})$ \\
\hline \hline Pitch of the lattice $(\Lambda)$ & 1.00 \\
Air hole diameter of inner core clad $\left(\mathrm{d}_{1}\right)$ & 0.84 \\
Air hole diameter of outer core $\left(\mathrm{d}_{2}\right)$ & 0.34 \\
Air hole diameter of outer core clad $\left(\mathrm{d}_{3}\right)$ & 0.90 \\
\hline \hline
\end{tabular}

The mode field distribution of the simple fiber at the wavelength of $1.55 \mu \mathrm{m}$ and the effective index of the fiber for the fundamental mode are presented in Figs. 3 and 4, respectively. The simple structure of the fiber has a negative dispersion only in a narrow wavelength range and is capable of dispersion compensation presented in Fig. 5.

Now, elliptical holes are placed instead of the circular holes in the first ring of the inner core clad and the dispersion profile is calculated for the new structure. Figure 6 shows the mode field distribution of the proposed fiber at the wavelength of $1.55 \mu \mathrm{m}$ and Fig. 7 shows the effective index of the fiber for the fundamental mode.

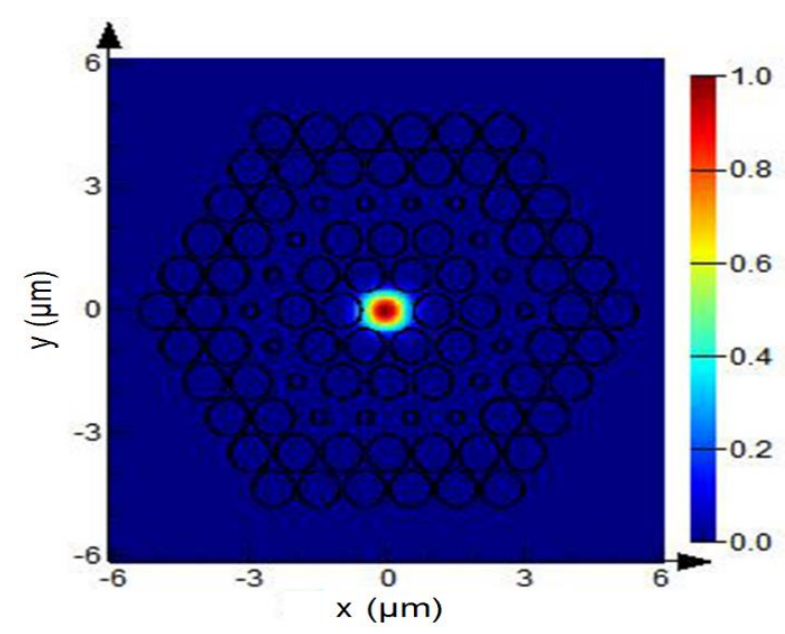

Fig. 3. The mode field distribution of the simple fiber at the wavelength of $1.55 \mu \mathrm{m}$.

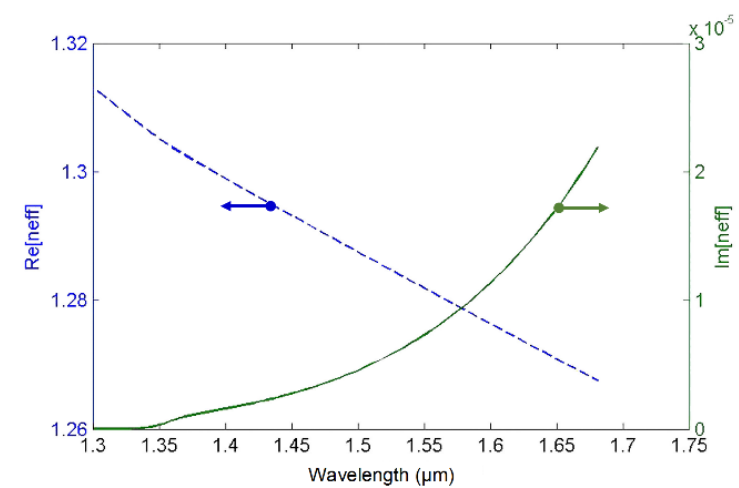

Fig. 4. The effective index of the simple hexagonal lattice wideband dispersion compensating fiber.

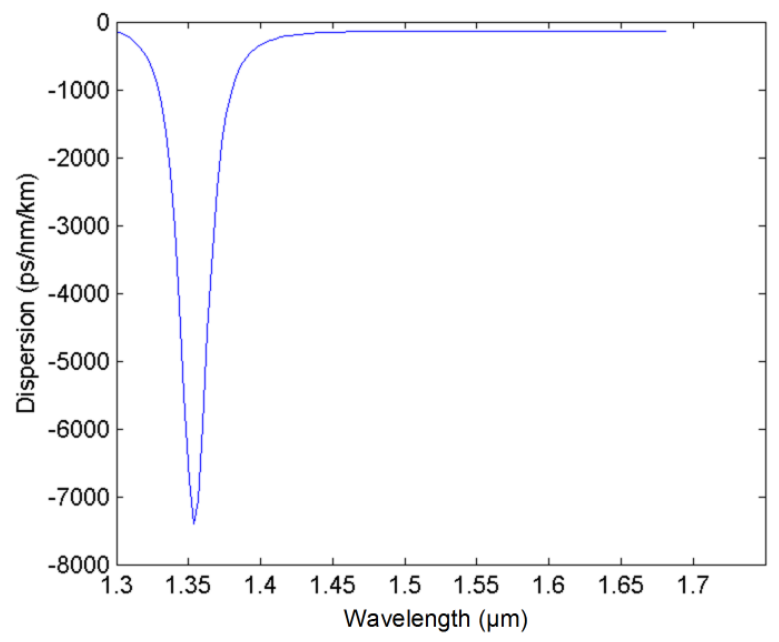

Fig. 5 The dispersion coefficient curve of the simple hexagonal lattice wideband dispersion compensating fiber. 
The curve of the dispersion coefficient Fig. 8 shows that when elliptical holes are placed instead of the circular holes causes the dispersion profile of the fiber to broaden and makes the fiber capable of dispersion compensation in a wider wavelength range compared to the corresponding simple structure. This fiber is capable of dispersion compensation throughout the $\mathrm{E}$ to $\mathrm{U}$ telecommunication bands. The minimum dispersion is -1006 $\mathrm{ps} /(\mathrm{nm} . \mathrm{km})$ at the wavelength of $1.68 \mu \mathrm{m}$, and the dispersion coefficient is $-710 \mathrm{ps} /(\mathrm{nm} . \mathrm{km})$ at the wavelength of $1.55 \mu \mathrm{m}$.

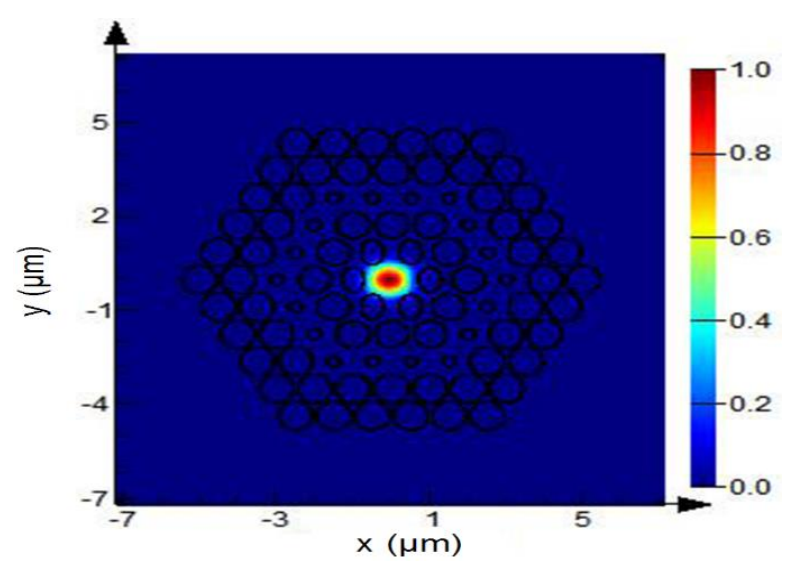

Fig. 6. The mode field distribution of the proposed fiber at the wavelength of $1.55 \mu \mathrm{m}$.

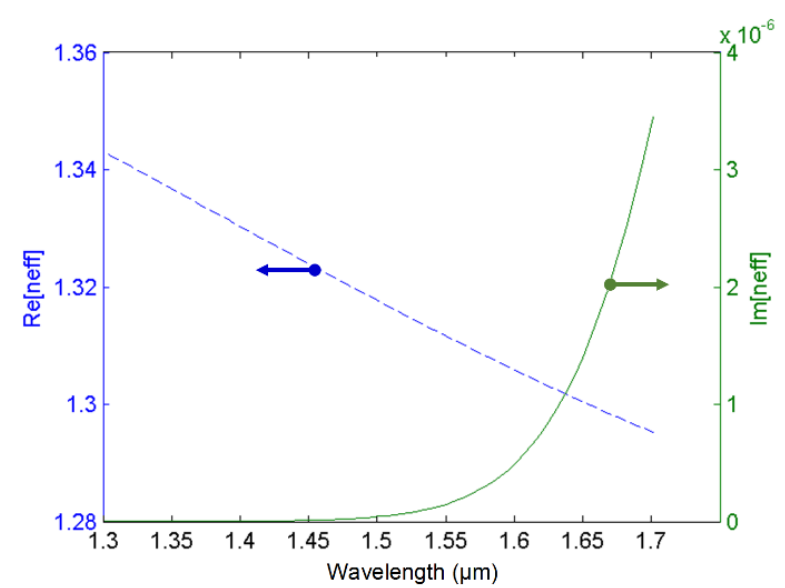

Fig. 7. The effective index curve of the proposed dispersion compensating fiber based on wavelength.

Figure 9 shows the influence of the changes in the diameter of elliptical holes in the $\mathrm{Y}$ axis on the dispersion profile of the fiber. As the diameter of elliptical holes in the $\mathrm{Y}$ axis decreases, the dispersion profile gets more positive and the minimum dispersion gets closer to the higher wavelengths. As the diameter of elliptical holes in the $\mathrm{Y}$ axis increases, the dispersion profile gets more negative and the minimum dispersion gets closer to the smaller wavelengths and in fact the dispersion profile gets narrower.

Figure 10 shows the influence of the changes in the diameter of elliptical holes in the $\mathrm{X}$ axis on the dispersion profile of the fiber. It can be seen that as the diameter of elliptical holes in the $\mathrm{X}$ axis decreases, the dispersion profile gets more positive and the minimum dispersion gets closer to the higher wavelengths. As the diameter of elliptical holes in the $\mathrm{X}$ axis increases, the dispersion profile gets more negative and the minimum dispersion gets closer to the smaller wavelengths.

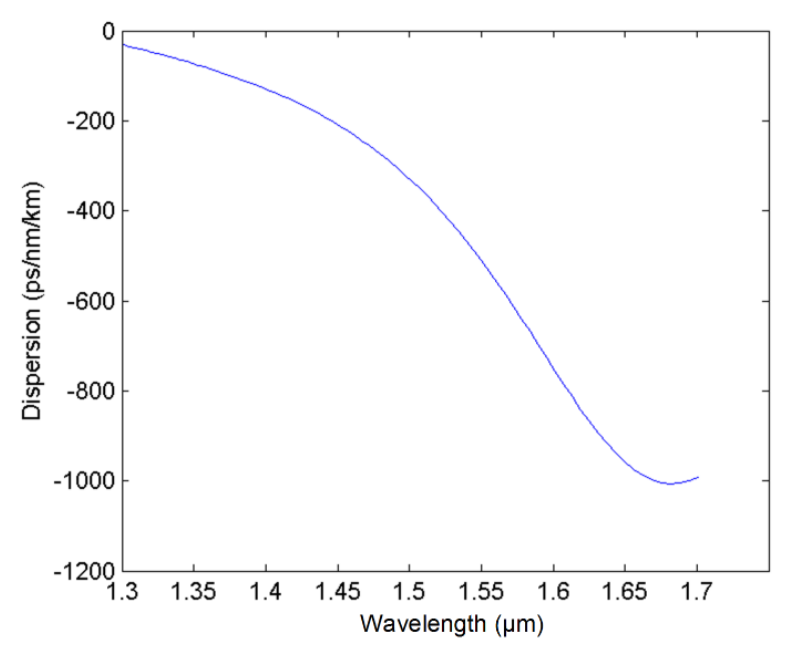

Fig. 8. The dispersion coefficient curve of the proposed dispersion compensating fiber.

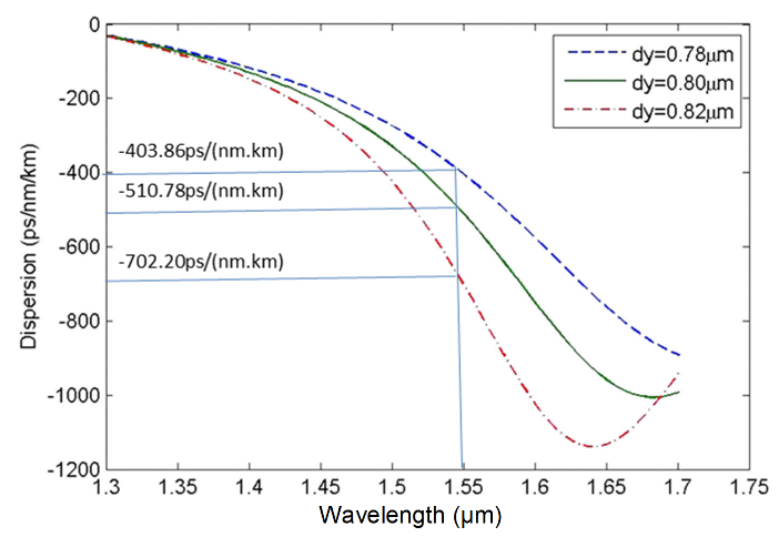

Fig. 9. The influence of the variation of the diameter of elliptical holes in the Y direction on the dispersion profile of the proposed fiber. 
To create the second core, instead of reducing the diameter of the holes in a ring, one can use another material in making a ring. To do so, in the structure of Fig. 1, instead of reducing the diameter of holes, fluorine-doped silica rods (1 mole \%) is used which is shown in Fig. 11. The characteristics and parameters of this structure are presented in Table 3.

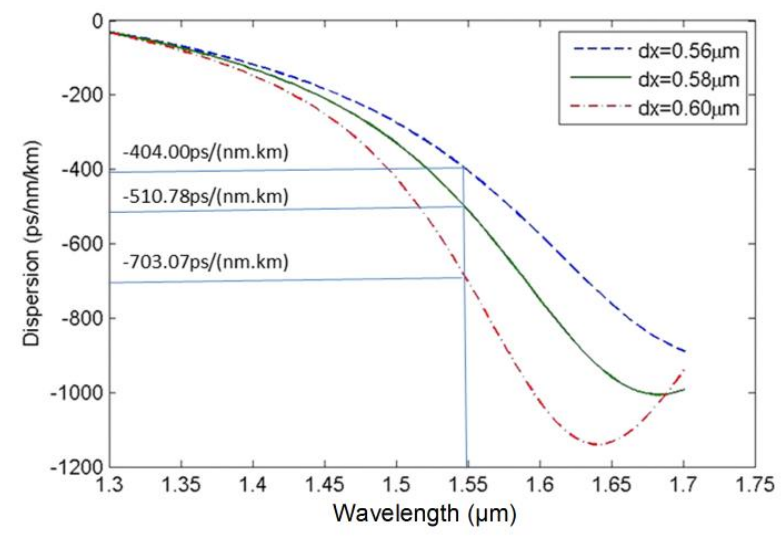

Fig. 10. The influence of the changes in the diameter of elliptical holes in the $\mathrm{X}$ axis on the dispersion profile of the proposed fiber.

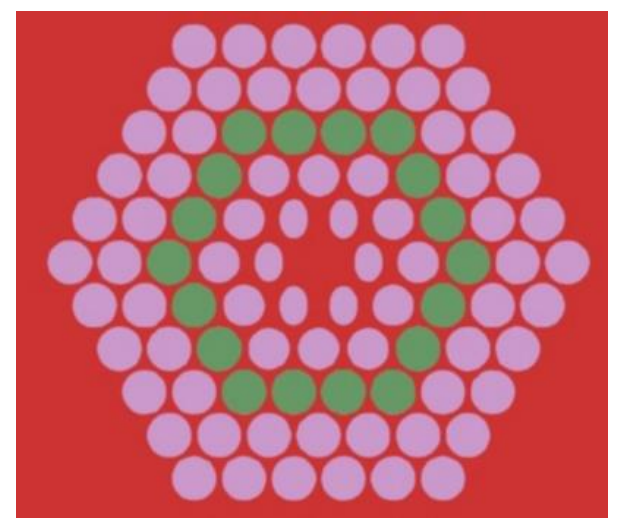

Fig. 11. The dispersion compensating fiber with elliptical holes and fluorine-doped silica rods.

Table 3. The parameters of fiber with elliptical holes and fluorine-doped silica rods.

\begin{tabular}{lc}
\hline \hline Parameter & Value $(\mu \mathrm{m})$ \\
\hline \hline Pitch of lattice $(\Lambda)$ & 1.00 \\
Air hole diameter of inner core clad $\left(\mathrm{d}_{1}\right)$ & 0.84 \\
Air hole diameter of outer core $\left(\mathrm{d}_{2}\right)$ & 0.90 \\
Air hole diameter of outer core clad $\left(\mathrm{d}_{3}\right)$ & 0.90 \\
The diameter of the ellipse in X axis $\left(\mathrm{d}_{\mathrm{x}}\right)$ & 0.29 \\
The diameter of the ellipse in Y axis $\left(\mathrm{d}_{\mathrm{y}}\right)$ & 0.40 \\
\hline \hline
\end{tabular}

The dispersion profile of the fiber is presented in Fig. 12. As one can see, the minimum dispersion is transmitted to the shorter wavelengths but this fiber still has a negative dispersion in a wide wavelength range and is capable of dispersion compensation. Additionally, the dispersion coefficient of the fiber is reduced. This fiber has a dispersion coefficient of $-113 \mathrm{ps} /(\mathrm{nm} . \mathrm{km})$ at the wavelength of $1.55 \mu \mathrm{m}$.

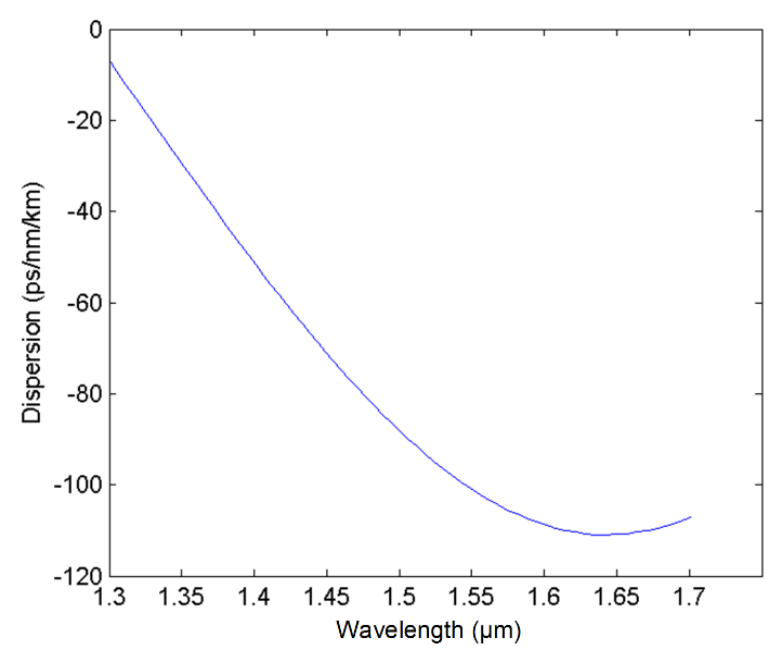

Fig. 12. The curve of dispersion compensating fiber with fluorine-doped silica rods.

\section{IV.CONCLUSION}

In this paper, a new structure was presented for dispersion compensating photonic crystal fibers to broaden the dispersion profile and increase the capability of dispersion compensation in a wide wavelength range. Placing elliptical holes in the first ring of the inner core clad of a dispersion compensating fiber of the hexagonal lattice caused an increase in the wavelength range of dispersion compensation and made the fiber have the capability of dispersion compensation throughout the $\mathrm{E}$ to $\mathrm{U}$ telecommunication bands. The minimum dispersion is -1006 $\mathrm{ps} /(\mathrm{nm} . \mathrm{km})$ at the wavelength of $1.68 \mu \mathrm{m}$, and the dispersion coefficient is $-710 \mathrm{ps} /(\mathrm{nm} . \mathrm{km})$ at the wavelength of $1.55 \mu \mathrm{m}$. Also, the influence of creating an outer core using the fluorine-doped silica rods (1 mole \%) was investigated in this study. By putting the fluorine-doped silica rods in the outer core, the minimum dispersion coefficient was transmitted a little to the smaller wavelengths but the fiber still has a negative dispersion in a wide wavelength range and is capable of dispersion compensation. Additionally, the 
dispersion coefficient of the fiber reduced. The dispersion coefficient is $-113 \mathrm{ps} /(\mathrm{nm} . \mathrm{km})$ at the wavelength of $1.55 \mu \mathrm{m}$.

\section{REFERENCES}

[1] M. Sadeghi, S. Olyaee, and F. Taghipour, "Design of low-dispersion fractal photonic crystal fiber," IJOP, vol. 6, pp. 57-64, 2012.

[2] F. Koohi-Kamali, M. Ebnali-Heidari, and M. K. Moravvej-Farshi, "Designing a dual-core photonic crystal fiber coupler by means of microfluidic infiltration,” Int. J. Opt. Photon. (IJOP), vol. 6, pp. 83-96, 2012.

[3] J. C. Knight, "Photonic crystal fibers," Nature, vol. 424, pp. 847- 851, 2003.

[4] J. C. knight, T. A. Birks, P. St. J. Russell, and D. M. Aktin, "All silica single-mode optical fiber with photonic crystal cladding," Opt. Lett., vol. 21, pp. 1547-1549, 1996.

[5] S. Kim and C. S. Kee, "Dispersion properties of dual-core photonic quasicrystal Fiber," Opt. Express, vol. 17, pp. 15885-15890, 2009.

[6] K. Thyagarajan, R. K. Varshney, P. Palai, A. K. Ghatak, and I. C. Goyal, "A novel design of a dispersion compensating fiber," IEEE Photon. Technol. Lett., vol. 8, pp. 1510-1512, 1996.

[7] J. L. Auguste, R. Jindal, J. M. Blondy, M. Clapeau, J. Marcou, B. Dussardier, G. Monnom, D. B. Ostrowsky, B. P. Pal, and K. Thygarajan, "-1800 ps/(nm.km) chromatic dispersin of $1.55 \mathrm{~mm}$ in dual concentric Core fibre," Electron. Lett. vol. 36, pp. 1689-1691, 2000.

[8] M. Aliramezani and Sh. Mohammad Nejad, "Numerical analysis and optimization of a dual-concentric-core photonic crystal fiber for broadband dispersion compensation," Opt. Laser Technol. vol. 42, pp. 1209-1217, 2010.

[9] B. L. Wang and J. M. Hsu, "Broadband dispersion compensating photonic crystal fiber with a high compensation ratio," Int. Conf. Advanced Materials for Science and Engineering (ICAMSE), pp. 471 - 474, 2016.

[10]J. Yuan, X. Sang, C. Yu, C. Jin, X. Shen, G. Zhou, S. Li, and L. Hou, "Large negative dispersion in dual-concentric-core photonic crystal fiber with hybrid cladding structure based on complete leaky mode coupling,"
Opt. Commun., vol. 284, pp. 5847-5852, 2011.

[11]P. S. Maji and P. R. Chaudhuri, "Design of ultra large negative dispersion PCF with selectively tunable liquid infiltration for dispersion compensation," Opt. Commun., vol. 325, pp. 134-143, 2014.

[12] S. Yang, Y. Zhang, X. Peng, Y. Lu, and S. Xie, "Theoretical study and experimental fabrication of high negative dispersion photonic crystal fiber with large area mode field," Opt. Express, vol. 14, pp. 3015-3023, 2006.

[13]T. Fujisawa, K. Saitoh, K. Wada, and M. Koshiba, "Chromatic dispersion profile optimization of dual-concentric-core photonic crystal fibers for broadband dispersion compensation," Opt. Express, vol. 14, pp. 893-900, 2006.

[14]T. Matsui, K. Nakajima, and I. Sankawa, "Dispersion compensation over all the telecommunication bands with doublecladding photonic-crystal fiber," J. Lightw. Technol. vol. 25, pp. 757-762, 2007.

[15] M. Selim Habib, M. Samiul Habib, M. I. Hasan, S. M. A. Razzak, R. R. Mahmud, and Y. Namihira, "Microstructure holey fibers as wideband dispersion compensating media for high speed transmission system," Optik, vol. 124, pp. 4984-4988, 2013.

[16]R. S. Quimby, Photonics and Lasers an Introduction, Wiley-Interscience, 2006.

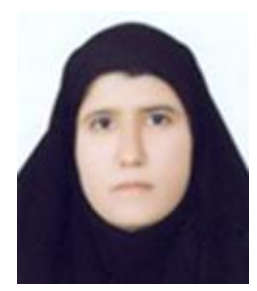

Esmat Jafari was born in Zahedan, Iran, in 1990. She received the B.S. degree in electronic engineering from the University of Sistan and Baluchestan, Zahedan, Iran, in 2012 and the M.S. degree in electronic engineering from the University of Sistan and Baluchestan, Zahedan, Iran, in 2015. She is currently working in the field of dispersion compensation photonic crystal fibers. Her 
research interests include optical devices and photonic crystals.

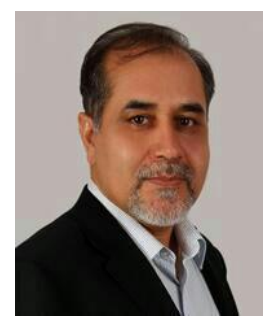

Mohammad of Sistan and Baluchestan, Zahedan, Iran, in 1986, the M.S. degree from the University of Tehran, Iran, in 1991, and the Ph.D. degree from Trabiat Modares University, Tehran, Iran, in 2008, all in electrical engineering. He then joined the University of Sistan and Baluchestan. As an Associate Professor with the University of Sistan and Baluchestan, his research areas are photonics, optoelectronics, analog integrated circuits, and plasmonics. He has served as a reviewer for a number of journals and conferences. 
THIS PAGE IS INTENTIONALLY LEFT BLANK. 